\title{
Laboratory simulation of solar magnetic flux rope eruptions
}

\author{
S. K. P. Tripathi ${ }^{1}$ and W. Gekelman ${ }^{2}$ \\ Physics \& Astronomy, University of California at Los Angeles, \\ Los Angeles, California 90095, USA \\ emails: ${ }^{1}$ tripathi@physics.ucla.edu and ${ }^{2}$ gekelman@physics.ucla.edu
}

\begin{abstract}
A laboratory plasma experiment has been constructed to simulate the eruption of arched magnetic flux ropes (AMFRs e.g., coronal loops, solar prominences) in an ambient magnetized plasma. The laboratory AMFR is produced using an annular hot $\mathrm{LaB}_{6}$ cathode and an annular anode in a vacuum chamber which has additional electrodes to produce the ambient magnetized plasma. Two laser beams strike movable carbon targets placed behind the annular electrodes to generate controlled plasma flows from the AMFR footpoints that drives the AMFR eruption. The experiment operates with a $0.5 \mathrm{~Hz}$ repetition rate and is highly reproducible. Thus, time evolution of the AMFR is recorded in three-dimensions with high spatio-temporal resolutions using movable diagnostic probes. Experimental results demonstrate outward expansion of the AMFR, release of its plasma to the background, and excitation of fast magnetosonic waves during the eruption.
\end{abstract}

Keywords. magnetic flux rope, prominence, coronal loop, coronal mass ejections, flares

\section{Introduction}

Arched Magnetic flux ropes (AMFRs) are arched magnetic structures that confine plasma and carry electrical current along its curved axis. The current generates twist in the AMFR magnetic field. Coronal loops, prominences, and filaments are main examples of solar AMFRs that have both footpoints anchored underneath the photosphere (Lang 2001). Solar AMFRs are observed to remain stable for many Alfvén transit times (time required for the Alfvén wave to travel from one footpoint to the other) in traditional astronomical and spacecraft observations (Abbot 1911). The persistent appearance of solar AMFRs for such a long duration (lasting days to weeks) implies that the current carried by solar AMFRs during this stable phase is well below the current threshold required for the kink instability. The stable phase of the solar AMFRs ends when instabilities that significantly affect the force balance of the AMFRs appear. The kink instability is a prime example of one such instability (Hood 1979). Several other possibilities include sausage instability, interaction of the AMFR with another AMFR, and changes in the ambient plasma conditions (Antiochos 1998 and Hansen 2004). Following the appearance of the instability, the AMFR erupts and frequently evolves into energetic events such as solar flares and coronal mass ejections (CMEs) [e.g., Dennis \& Schwartz (1989), Chen (1996), Cremades \& Bothmer (2004), and Hildner (1975)].

Technological developments in solar observations have drastically improved the measurement of the global properties of the solar AMFRs. However, it is still not feasible to directly measure the internal magnetic field and other important plasma parameters of the solar AMFRs with a good spatial and temporal resolutions. This severely limits our capability to experimentally test the validity of the theoretical models and identify the important microphysics that may not be captured in numerical simulations. Laboratory 


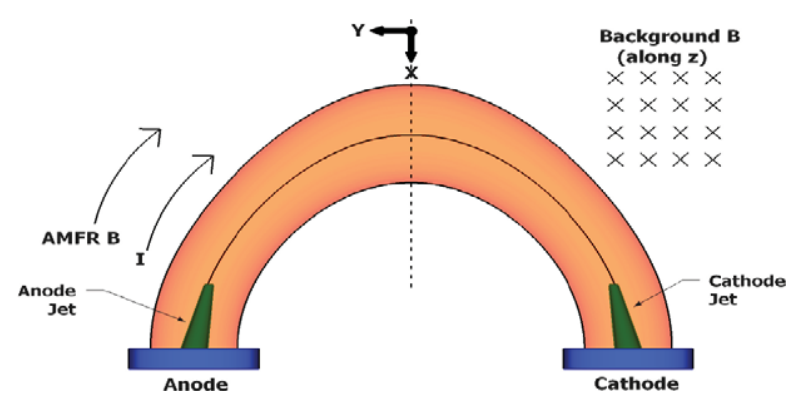

Figure 1. Schematic showing the experimental arrangement for simulating the solar AMFR eruptions. Laser generated plasma jets, relative directions of the AMFR and background magnetic fields, AMFR current I, Cartesian coordinates $x y z$, and both electrodes are indicated.

experiments that produce carefully scaled models of actual solar AMFRs have started playing an important role in bridging this gap (Alexander 2007, Tripathi, Bellan, \& Yun 2007, and Tripathi \& Gekelman 2010).

The conventional laboratory technique for producing an AMFR utilizes a magnetized arc plasma source which produces an AMFR containing several kilo-ampere electrical current (Hansen 2004). The current builds up within few microseconds and greatly exceeds the threshold for the kink instability. Hence, the stable phase of the AMFRs is not observed in these experiments. Moreover, the AMFRs in conventional experiments evolve in a vacuum environment unlike actual solar environment where magnetized plasma exists in the ambiance. Thus, a non-conventional approach is needed to simulate the impulsive eruption of a stable solar AMFR. In this article, we describe a new laboratory plasma experiment that utilizes three high-repetition-rate plasma sources to generate the AMFR, an ambient plasma, and controlled plasma flows from the AMFR footpoints.

\section{Experimental setup}

The AMFR is produced in a $4.0 \mathrm{~m}$ long (1.0 m diameter) vacuum chamber that has electromagnets to produce a $25 \mathrm{G}$ magnetic field along its axis. A hot $\mathrm{LaB}_{6}$ cathode $(20$ $\mathrm{cm} \times 20 \mathrm{~cm}, 1700{ }^{\circ} \mathrm{C}$ ) and a wire-mesh anode are mounted at one end of the chamber to produce an ambient argon plasma (neutral gas pressure $=5.0 \times 10^{-4}$ torr, plasma density $n=2.0 \times 10^{12} \mathrm{~cm}^{-3}$, electron temperature $\mathrm{T}_{e} \sim 4 \mathrm{eV}$, pulse width $=20 \mathrm{~ms}$, repetition rate $=0.5 \mathrm{~Hz}$ ). A Cartesian coordinate system $x y z$ with the $z$ direction oriented along the axis of the vacuum chamber (towards the rectangular cathode) is used to delineate the relative position of the AMFR plasma source in the ambient plasma. As shown in Fig. 1 , the symmetry plane of the AMFR is a $x y$ plane at $z=0 \mathrm{~cm}$. The AMFR is produced using an additional set of an annular disk-shaped anode and a hot $\mathrm{LaB}_{6}$ cathode $(7.6 \mathrm{~cm}$ outer and $1.3 \mathrm{~cm}$ inner diameter) mounted inside the vacuum chamber on two radially movable shafts. The AMFR footpoints are separated by $23 \mathrm{~cm}$. Each AMFR electrode is surrounded by an electromagnet to generate an arched magnetic field ( $B \sim 1 \mathrm{kG}$ at the AMFR footpoints). The AMFR magnetic field makes a $90^{\circ}$ angle from the magnetic field of the ambient plasma. Using this arrangement, highly reproducible AMFRs $\left(n \sim 10^{13}\right.$ $\mathrm{cm}^{-3}, \delta n / n<0.005, \mathrm{~T}_{e} \sim 4 \mathrm{eV}$, pulse width $\left.=2.0 \mathrm{~ms}, I=42 \mathrm{~A}\right)$ are produced in the afterglow of the every ambient plasma pulse. During each pulse, a stable AMFR is formed $\sim 150 \mu$ s after application of the voltage on the AMFR electrodes. Figure 2(a) shows a fast camera image of the stable AMFR. The persistent appearance of the images and a stationary density profile characterize the stable phase of the AMFR.

Two infrared laser beams (1064 nm, $E \sim 0.8 \mathrm{~J} /$ pulse) are triggered in the middle of the AMFR pulse to ablate two carbon rods placed behind the annular electrodes. The 

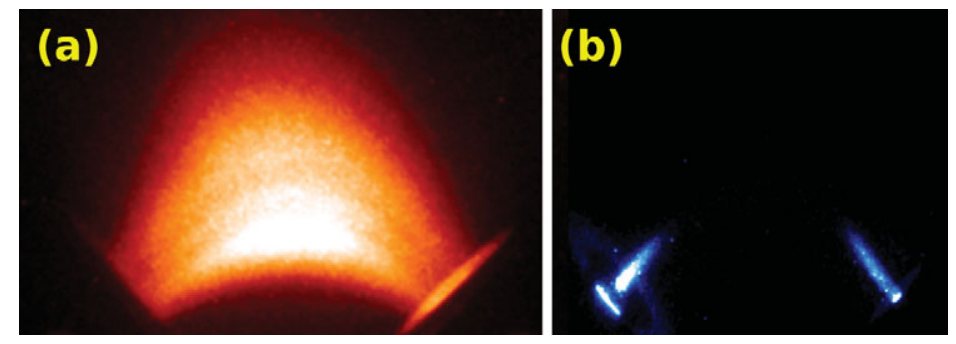

Figure 2. (a) An unfiltered fast camera image of the stable argon AMFR before the laser trigger and (b) a $460 \mathrm{~nm}$ narrow-passband filtered image of the carbon jets at $0.24 \mu$ s after the laser trigger. The narrow-passband filter transmits radiation from the carbon jets but blocks visible radiation from the argon AMFR.

laser-ablated carbon generates plasma jets from both AMFR footpoints. Following the laser trigger, both rods are moved by a small step using computer-controlled motors to ensure that a fresh target surface is ablated at every shot and a good reproducibility is maintained in the experiment. A fast camera image of the laser jets [see Fig. 2(b)] in the AMFR was acquired using a $460 \mathrm{~nm}$ passband filter which shows that the jets are composed of $\mathrm{C}^{++}$ions. The jets propagate with a supersonic velocity $\left(v \sim 5.0 \times 10^{4} \mathrm{~m} / \mathrm{s}\right)$ in the AMFR (ion acoustic speed $c_{s} \sim 5.0 \times 10^{3} \mathrm{~m} / \mathrm{s}$ ). The AMFR current following the appearance of the jets increases to $\sim 600 \mathrm{~A}$ which is above the kink instability threshold. Since the experiment is highly reproducible and runs at $0.5 \mathrm{~Hz}$ repetition rate, computercontrolled movable probes are employed to collect the magnetic field and the density data with a high spatio-temporal resolutions ( $\Delta t=20 \mathrm{~ns}, \Delta x=1-5 \mathrm{~mm}$ ).

\section{Results and Discussion}

The density profile of the AMFR is presented in Fig. 3 at four distinct times to show the evolution of the AMFR following the laser trigger $(t=0 \mu \mathrm{s})$. The profile has been

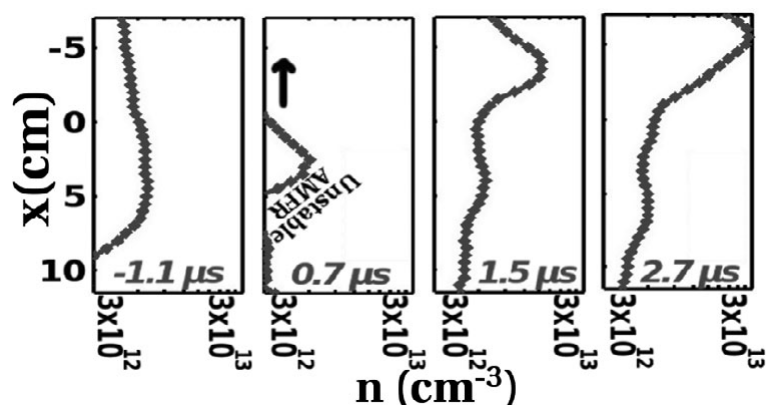

Figure 3. The AMFR density profiles $n(x)$ are shown at four distinct times. The outward motion of the AMFR can be seen as motion of the density peak along the $-x$ direction.

measured along the dashed $x$ line shown in Fig. 1 at $y=0 \mathrm{~cm}$ and $z=-5 \mathrm{~cm}$. Collection of the data in the $z=0 \mathrm{~cm}$ plane is avoided since the laser beams propagate in this plane and they can easily destroy miniature diagnostic probes. As stated earlier, the laser generated jets significantly increase the AMFR current making it kink unstable. Moreover, the stable phase of the AMFR is maintained due to the balance of the outward hoop force and the inward forces including the magnetic tension force (Krall et al. 2000). The increase in the AMFR current significantly enhances the outward hoop force and the AMFR expands in the outward direction. The $t=-1.1 \mu$ s plot represents the stationary 
density profile of the stable AMFR. The AMFR becomes extremely dynamic following the laser trigger. The $t=0.7 \mu$ s plot shows that the AMFR plasma is released to its ambiance immediately following the appearance of the laser jets and it becomes thinner. The other two plots clearly show the outward motion of the AMFR. In our recent paper (see Tripathi \& Gekelman 2010), excitation of fast waves in the ambient plasma during the eruption of the laboratory AMFR has been described in detail alongwith changes in the magnetic structure of the AMFR.

These results demonstrate the important characteristics of solar AMFR eruptions such as its outward motion and ejection of the plasma to the ambiance. Camera images also show that the laboratory AMFRs resemble the structure of the solar AMFRs. Comparison of the plasma parameters of the laboratory and solar AMFRs are presented in Table 1. A MHD scaling of the solar AMFR parameters to the laboratory parameters requires

Table 1. Comparison of solar and laboratory plasma parameters

\begin{tabular}{llll}
\hline & Lower Corona & Coronal loops & Laboratory AMFR \\
\hline Density $n\left(\mathrm{~cm}^{-3}\right)$ & $\sim 10^{9}$ & $10^{9}-10^{11}$ & $\sim 10^{13}$ \\
Temperature $T(\mathrm{~K})$ & $\sim 10^{6}$ & $10^{6}-10^{7}$ & $\sim 10^{5}$ \\
Length L $(\mathrm{m})$ & $\sim 10^{8}-10^{9}$ & $\sim 10^{7}$ & 0.51 \\
Magnetic field B $(\mathrm{G})$ & $\sim 10$ & 20 & 1000 \\
Plasma $\beta$ & & $10^{-2}-10^{-1}$ & $10^{-3}-10^{-1}$ \\
Lundquist number S & $10^{13}$ & $10^{2}-10^{4}$ \\
\hline
\end{tabular}

that a large Lundquist number and low plasma $\beta$ (ratio of plasma pressure and magnetic field pressure) are retained in the experiment. The results indicate that the laboratory AMFR has appropriate values to simulate the actual solar AMFRs. High resolution measurements of the magnetic field, density and electron temperature are planned in near future to further explore the details of the AMFR eruption in our experiment.

\section{Acknowledgment}

This experiment was performed at the Basic Plasma Science Facility (BaPSF) at University of California, Los Angeles and was jointly supported by US DOE and NSF.

\section{References}

Abbot, C. G. Sep 1911, The Sun (D. Appleton and Company, New York and London), p. 128-182 Alexander, D. 2007, ApשSSS 307, 197

Antiochos, S. K. 1998, ApJ,502, L181

Chen, J. 1996, J. Geophys. Res. 101, 27499

Cremades H. \& Bothmer, V. 2004, A $\mathscr{S} A$ 422, 307

Dennis, B. R. \& Schwartz, R. A. 1989, Solar Phys. 1989, 121, 75

Hansen, J. F. \& Bellan, P. M. 2001, ApJ, 563, L183

Hansen, J. F., Tripathi, S. K. P., \& Bellan, P. M. 2004, Phys.Plasmas 11, 3177

Hildner, E. et al. 1975, Solar Phys. 42, 163

Hood, A. W. \& Priest, E. R. 1979, Solar Phys., 64, 303

Krall, J., Chen J., \& Santoro R. 2000, ApJ, 539, 964

Lang, K. R. 2001, The Cambridge Encyclopedia of the Sun (Cambridge University press), 1st Ed. p. 106-143

Tripathi, S. K. P., Bellan, P. M., \& Yun, G. S. 2007, Phys. Rev. Lett., 98, 135002

Tripathi, S. K. P. \& Gekelman, W. 2010, Phys. Rev. Lett., 105, 075005 